\title{
Effect of Heat Stress on the Expression of GABA Receptor mRNA in the HPG Axis of Wenchang Chickens
}

-Author(s)

\section{Xie LJ' \\ Xie $X Z^{\prime}$ \\ Zhou YW \\ Liang $\mathrm{C}^{\prime}$ \\ Jiang $Y Y$ \\ Chen $Z^{\prime}$}

Ministry of Education Key Laboratory for Tropical Animal and Plant Ecology, Hainan Normal University, Haikou, 571158, China

\section{Mail Address}

Corresponding author e-mail address Z Chen

College of Life Sciences, Hainan Normal University, Longkun South Road, Haikou, 571158, China.

Phone: +86-898-65883521

Fax: $\quad+86-898-65888715$

Email: zh.chen@hainnu.edu.cn

\section{- Keywords}

Heat Stress; GABA receptor mRNA; HPG axis; Chickens.

\section{ABSTRACT}

We investigated the effect of heat stress (HS) on the expression of the GABA receptor in the hypothalamic-pituitary-gonadal (HPG) axis of Wenchang chickens. Real-time quantitative RT-PCR (qRT-PCR) was used to quantify the GABA receptor mRNA levels along the HPG axis of chickens under $\mathrm{HS}\left(40 \pm 0.5^{\circ} \mathrm{C}\right)$ for $1-6$ weeks. Our results showed that the expression of $\mathrm{GABA}_{A}$ and $\mathrm{GABA}_{B}$ receptor at the mRNAs levels in the tissues of HPG axis exhibited fluctuation and variability. After $H S$, the mRNA level of $G_{A B A}$ receptor was significantly reduced in the hypothalamus of 1-week-old and in the pituitary of 3-week-old chickens, but significantly increased in the pituitary of 1-, 4-, and 5-weekold chickens. The $G A B A_{B}$ receptor mRNA level significantly declined in the hypothalamus of 1-week-old and in the pituitary of 3-week-old chickens, but was significantly upregulated in the pituitary and testis of 1- and 2-week-old chickens. At other time points, the expressions of $G_{A B A}$ receptor and $G A B A_{B}$ receptor showed no significant differences compared with control group. These results indicated that the levels of $G_{A B A}$ receptor and $G A B A_{B}$ receptor mRNAs varied in different tissues of the HPG axis in chickens of different ages, displaying temporal and spatial variations. GABA receptor behaved as a positively-regulated gene by HS, i.e., its mRNA was increased by HS; similarly, it was a negativelyregulated gene by HS, when its expression was reduced by HS.

\section{INTRODUCTION}

Heat stress caused by high ambient temperatures adversely affects the growth, development, and reproduction of animals (Rensis \& Scaramuzzi, 2003; McMichael \& Dear, 2010). It is also one of the main factors restricting the development of intensive large-scale poultry farming. HS affects the behavior, physiology, and gene expression of animals. Under HS, chickens show behavioral changes, including reduced food intake, extended wing-spreading resting, increased water intake, etc. Under high temperature, the bird's body resists to HS by inducing the release of hypothalamic-pituitary hormones, i.e., they regulate their physiological activities, such as metabolism, immunity, and reproduction, etc. via synthesis and release of hormones related to the adrenal axis, thyroid axis, and gonadal axis (Akazome et al., 2002; Elnagar et al., 2010; Sohail et al., 2010; Yin et al., 2011). HS induces the expression of numerous genes, such as heat shock protein 70, growth- and metabolism-related genes (EGFR, SFRP1, and PDK4), immune-related genes (HSF1, $B C l-2$, and $B a x-\alpha$ ), reproductive hormone receptor-associated genes (LHR, ESR, PRLR, and FSHR), rhythmic genes (Per2), etc. (Cai et al., 2004; Zhao et al., 2012; Li \& Cui, 2013).

To date, the impact of HS on the expression of the GABA (gammaaminobutyric acid) receptor has not been reported. As an inhibitory 
neurotransmitter, GABA is widely present in the central nervous system and peripheral tissues; it can promote food intake in animals, regulate endocrine, autoimmune, hormonal, and reproductive physiology, and improve functions of anti-hypoxia, anti-HS, etc. in animals (Chen et al., 2001; Jonaidi et al., 2002; Chen et al., 2014; Chen et al., 2015). GABA exerts its biological functions via interaction with GABA receptor. Our previous studies showed that prenatal HS significantly increased the density of the GABA receptor protein on the membrane of the testicular and ovarian cells of newborn mice. HS also reduced the positive rate and average densitometric value of the $\mathrm{GABA}_{\mathrm{B}}$ receptor in immunostaining, indicating a delayed development of $\mathrm{GABA}_{\mathrm{B}}$ receptor (Wang et al., 2011; Xie et al., 2011).

Due to the widespread use of fluorescent quantitative PCR (qPCR), the study of the differential expression of a trace amount of gene transcripts is fast and convenient. Knowing the expression pattern of $\mathrm{GABA}_{A}$ and $\mathrm{GABA}_{B}$ receptors along the HPG axis in chickens under HS not only provides a theoretical basis for better understanding of the development and the pathological analysis of reproductive system in chickens, but also a reference for intensive poultry farming during hot seasons. Therefore, in the present study, the differential expression of gamma-aminobutyric acid (GABA) A receptor, beta 3(NM_205346.3) and gammaaminobutyric acid (GABA) B receptor, 2(XM_419066.4) along the HPG axis of 1-to 6-weeks old Wenchang chickens was evaluated. The qRT-PCR technique was applied to determine the effects of changes in GABA receptor gene expression on the development of the gonadal axis of chickens and the effects of HS on its development.

\section{MATERIALS AND METHODS}

\section{Sample collection}

Healthy 1-day-old Wenchang chicks, provided by the Hainan Yongji Poultry Co., Ltd. (Hainan, China), were randomly divided into control (CK) and HS groups, with 140 chickens per group. In each group, there were 104 males, used for the collection of hypothalamus, pituitary, and testicular tissue samples, and 36 females, used for harvesting ovarian tissue samples.

Chickens of the HS group were daily placed between 12:00 and 14:00, in an artificial climate chamber at the temperature of $40 \pm 0.5^{\circ} \mathrm{C}$ and humidity of $70-80 \%$, while the CK birds were kept at room temperature. All birds had free access to water and feed (Chen et al., 2014). At 15:00 of each weekend of weeks 1-6, appropriate numbers of male and female birds in each group were sacrificed to collect hypothalamus, pituitary, testis, and ovary samples. In each group, triplicate samples of the hypothalamus and pituitary were collected from 3-10 males per group, and testis and ovary samples were collected from 6 birds per group. (Cheng et al., 2012). The tissue samples were frozen in liquid nitrogen immediately after the dissection and stored at $-70{ }^{\circ} \mathrm{C}$ until subsequent RNA extraction.

This experiment was approved by the Hainan Normal University Animal Experimentation Ethics Committee.

\section{Total RNA isolation and CDNA synthesis}

About 10-20 mg of respective hypothalamus, pituitary, testis, and ovary samples were used to isolate total RNAs using the RNAprep Animal RNA Purification Kit (Tiangen, DP431, Beijing, China). The purity, concentration, and integrity of the extracted RNAs were determined using a spectrometer and agarose gel electrophoresis. Total RNAs were then reversetranscribed into first strand CDNA using the FastQuant RT Kit (with gDNase) (Tiangen, KR106, Beijing, China). The cDNA products were stored in $-20^{\circ} \mathrm{C}$ freezer for further use.

\section{Real-time PCR}

The gene sequences of GABA receptors of Gallus gallus were retrieved from the NCBI database, and the $\mathrm{qPCR}$ primers were designed using the Primer Premier 5.0 software. The primers for $\mathrm{GABA}_{A}$ receptor B3 subunit gene (NM_205346.3) were as follows: forward, 5'-GCCCTCCATACTGATTACCAT-3'; reverse, 5'-GAATACAAAGCAGCCCATAAGATA-3'. The primers for GABA $_{B}$ receptor subunit GB2 (XM_419066.4) and internal control $\beta$-actin genes (NM_205518.1) were based on previous report (Cheng et al., 2012). The primers for $G A B A_{B}$ receptor subunit $G B 2$ were as follows: forward, 5'-TCGGGACCAACCCAACGTGC-3', reverse, 5'-CGTGC TGGCCTGATTGACGCT-3'. The primers for $\beta$-actin: forward, 5'-CCGCTCTATGA AGGCTACGC-3'; reverse, 5'-CTCTCGGCTGTGGTGGTGAA-3'.

The qPCR was set up using the Super Real PreMix Plus (SYBR Green) (Tiangen, FP205, Beijing, China) following the manufacturer's instructions, and the annealing temperature and the primer concentrations were optimized by experiment. The reaction $(20 \mu \mathrm{L})$ included $10 \mu \mathrm{L} 2 \times$ SuperReal PreMix Plus, $0.6 \mu \mathrm{L}$ of each forward and reverse primers $(10 \mu \mathrm{M})$, and $1 \mu \mathrm{L}$ CDNA, with volume adjusted with sterile double distilled $\mathrm{H}_{2} \mathrm{O}$. The amplification was conducted on the Roche 
LightCycler ${ }^{\circledR}$ Nano quantitative PCR instrument, and the PCR conditions were as follows: $95^{\circ} \mathrm{C}$ denaturation 15 min; $95^{\circ} \mathrm{C} 10 \mathrm{~s}, 60^{\circ} \mathrm{C} 20 \mathrm{~s}, 72{ }^{\circ} \mathrm{C} 20 \mathrm{~s}, 40$ cycles; with dissociation curve. Reactions added with sterile $\mathrm{H}_{2} \mathrm{O}$ instead of the template served as negative control, and each sample was with 3 replicates. After $P C R$, the dissociation curve was used to confirm the specific amplification, and a quantitative analysis was performed based on the Ct values of both the target and internal control genes.

\section{Data processing}

The relative quantification of gene expression was performed using $2^{-\Delta \mathrm{Ct}}$ method $\left[\Delta \mathrm{Ct}=\mathrm{Ct} \mathrm{t}_{\text {target gene }}-\mathrm{Ct}_{\text {reference }}\right.$ gene] (Jin et al., 2014). Data were statistically analyzed using Microsoft Excel and SPSS 16.0 software, and the relative expression was presented as means \pm SEM. The differences in gene expression between $\mathrm{CK}$ and $\mathrm{HS}$ groups were analyzed by Student $t$-test.

\section{RESULTS}

\section{The integrity of the extracted total RNA}

The $\mathrm{OD}_{260} / \mathrm{OD}_{280}$ values of the extracted total RNAs were between 1.8 and 2.1. The results of $1.5 \%$ agarose gel electrophoresis (Figure 1 ) indicated good RNA integrity, without degradation and other contaminants.

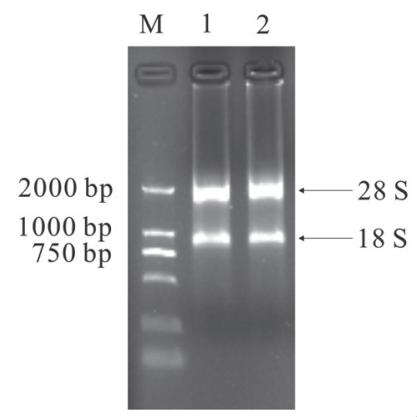

Figure 1 - Agarose gel electrophoresis of total RNAs. Note: M, Marker DL2000; 1 , ovarian RNA of the CK group in week 3; 2, ovarian RNA of the HS group in week 3. Agarose gel electrophoresis of other samples also clearly showed the $28 \mathrm{~s}$ and $18 \mathrm{~s}$ bands like these two representative samples.

\section{The sensitivity and reliability of qPCR}

The $\mathrm{qPCR}$ amplification curves of GABA $\mathrm{A}_{\mathrm{A}}$ receptor, $\mathrm{GABA}_{\mathrm{B}}$ receptor, and $\beta$-actin genes of the HPG axis displayed a S-shape, with smooth baseline and large slope rate in the exponential amplification phase. All dissociation curves showed a single peak, without non-specific amplification and primer dimers. The amplification efficiencies were close between both the target and control genes (both in the range of
0.9-1.1). The qPCR products were then cloned and subjected to sequencing, and the sequences showed $100 \%$ homology with the original sequence of Gallus gallus in GenBank.

\section{The effect of HS on the expression of GABA receptor in the HPG axis}

The relative expressions of $G_{A B A}$ receptor and $G_{A B A_{B}}$ receptor against the internal control $\beta$-actin gene in the hypothalamus, pituitary, testis, and ovary of chickens at different week-ages are shown in Figure 2 and 3.

As shown in Figure 2, the levels of GABA receptor mRNA in the tissues of HPG axis of 1- to 6-weekold chicken displayed fluctuation and variability. Its expression in the hypothalamus peaked in the $1^{\text {st }}$ week, declined in the $2^{\text {nd }}$ week, but exhibited a rising trend during weeks 3-6. The levels of $\mathrm{GABA}_{A}$ receptor mRNA in the pituitary increased in weeks 1-3, showed a decreasing trend in weeks 4-5, but increased in week 6 . Its expression in the testis exhibited a decreasing trend in the first 2 weeks, increased in the $3^{\text {rd }}$ week, declined in the $4^{\text {th }}$ week, but increased again at 5-6 weeks of age. The expression of $\mathrm{GABA}_{\mathrm{A}}$ receptor in the ovary showed a decreasing trend in the first 2 weeks, increased in weeks 3 and 5, but declined in weeks 4 and 6 . The GABA $A_{A}$ receptor mRNA in the testis and ovary displayed a similar trend of expression, and its maximum level in the pituitary, testicular, and ovarian tissues appeared in the week 3.

HS affected the expression of $G_{A B A_{A}}$ receptor. One week after $\mathrm{HS}$, the levels of $\mathrm{GABA}_{A}$ receptor mRNA in the hypothalamus significantly decreased 0.2 -fold relative to the control group, whereas its expression in the pituitary increased 3.4-fold relative to the control group. While the levels of $\mathrm{GABA}_{A}$ receptor mRNA in the pituitary of chickens significantly decreased 0.2fold of that of the control group three weeks after HS, its expression in the same tissue significantly increased 1.9-fold of that of the control group at 4 weeks of age. Five weeks after $\mathrm{HS}$, the expression of $\mathrm{GABA}_{\mathrm{A}}$ receptor in the pituitary of chickens significantly increased 3.9fold compared with the control group.

As shown in Figure 3, the expression of $G_{A B A}$ receptor in the tissues of HPG axis of 1- to 6-weekold chickens also varied and fluctuated. Its expression in the hypothalamus showed a decreasing trend in the first four weeks, increased in week 5 week, but decreased again in week 6 . The expression of GABA $A_{B}$ receptor mRNA in the pituitary increased in weeks 1-3, but declined in weeks 4-6. Its expression in the 

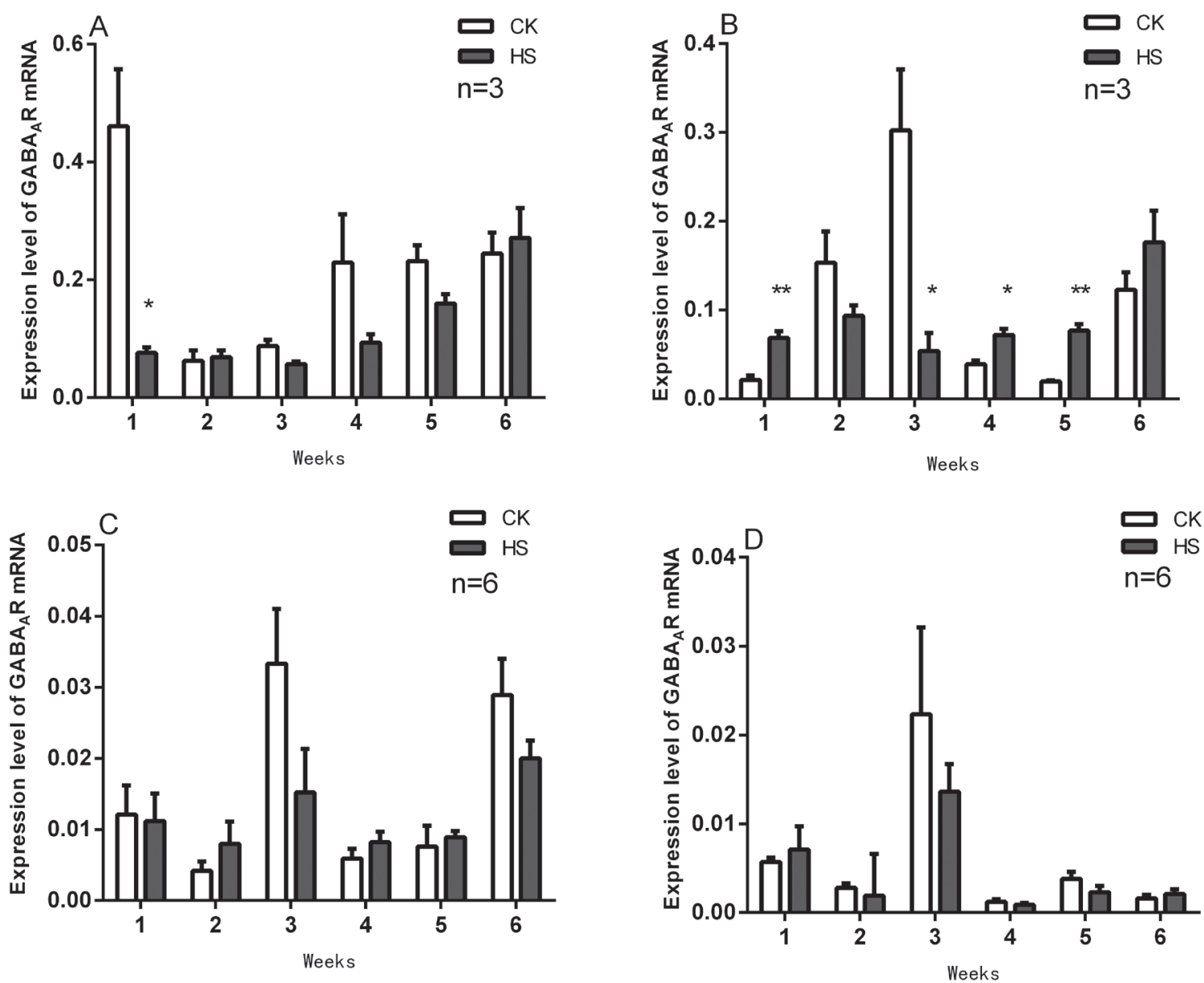

Figure 2 - Relative levels of GABAA receptor mRNA in the hypothalamus, pituitary, testis, and ovary of Wenchang chickens. Note: A, hypothalamus; B, pituitary; C, testis; and $D$, ovary. The results are expressed as mean \pm SEM by $2-\nabla C \mathrm{Ct} ;{ }^{*} \mathrm{p}<0.05$.

testis exhibited an increasing trend in the first three weeks, decreased in weeks 4-5, but increased again in week 6 . The levels of $G_{A B A}$ receptor mRNA in the ovary showed an increasing trend in the first three weeks and in week 5, but decreased in weeks 4 and 6 . The $G A B A_{B}$ receptor mRNA in the testis and ovary displayed a similar trend of expression during the first four weeks, and its maximum level of expression in the pituitary, testicular, and ovarian tissues appeared at 3 weeks of age.

HS affected the expression of $\mathrm{GABA}_{\mathrm{B}}$ receptor in chickens. One week after HS, the levels of GABA receptor mRNA in the hypothalamus and testis of chickens significantly decreased 0.4- and 0.3-fold, respectively, compared with the control group; however, its expression in the pituitary increased 1.7fold relative to the control group. While the levels of $G_{A B A}$ receptor mRNA in the pituitary and testis of chickens significantly increased 4.8- and 2.8-fold relative to the control group two weeks after HS, its expression in the pituitary significantly decreased 0.1 fold relative to the in control group at three weeks of age. Six weeks after HS, the expression of GABA receptor in the testis of chickens significantly dropped, and corresponded to 0.2 -fold of that in control group.

\section{DISCUSSION}

The present study detected low levels of $G_{A B A}$ receptor $\beta 3$ subunit and $G A B A_{B}$ receptor $R 2$ subunit mRNAs in the hypothalamus, pituitary, testicular, and ovarian tissue samples of Wenchang chickens. The expression of GABA receptor gene in different tissues of Wenchang chickens at different ages showed fluctuation and variability. Its expression in the hypothalamus and pituitary were slightly higher than those in the testis and ovary, which is different from previously reported results that the GABA contents in peripheral tissues, such as fallopian tubes, ovaries, testes, etc. were 2-fold higher than those in the central nervous system (Erdo et al., 1982).

One week after HS, the expression of both $\mathrm{GABA}_{\mathrm{A}}$ receptor and $G A B A_{B}$ receptor were significantly reduced in the hypothalamus, indicating that the normal 

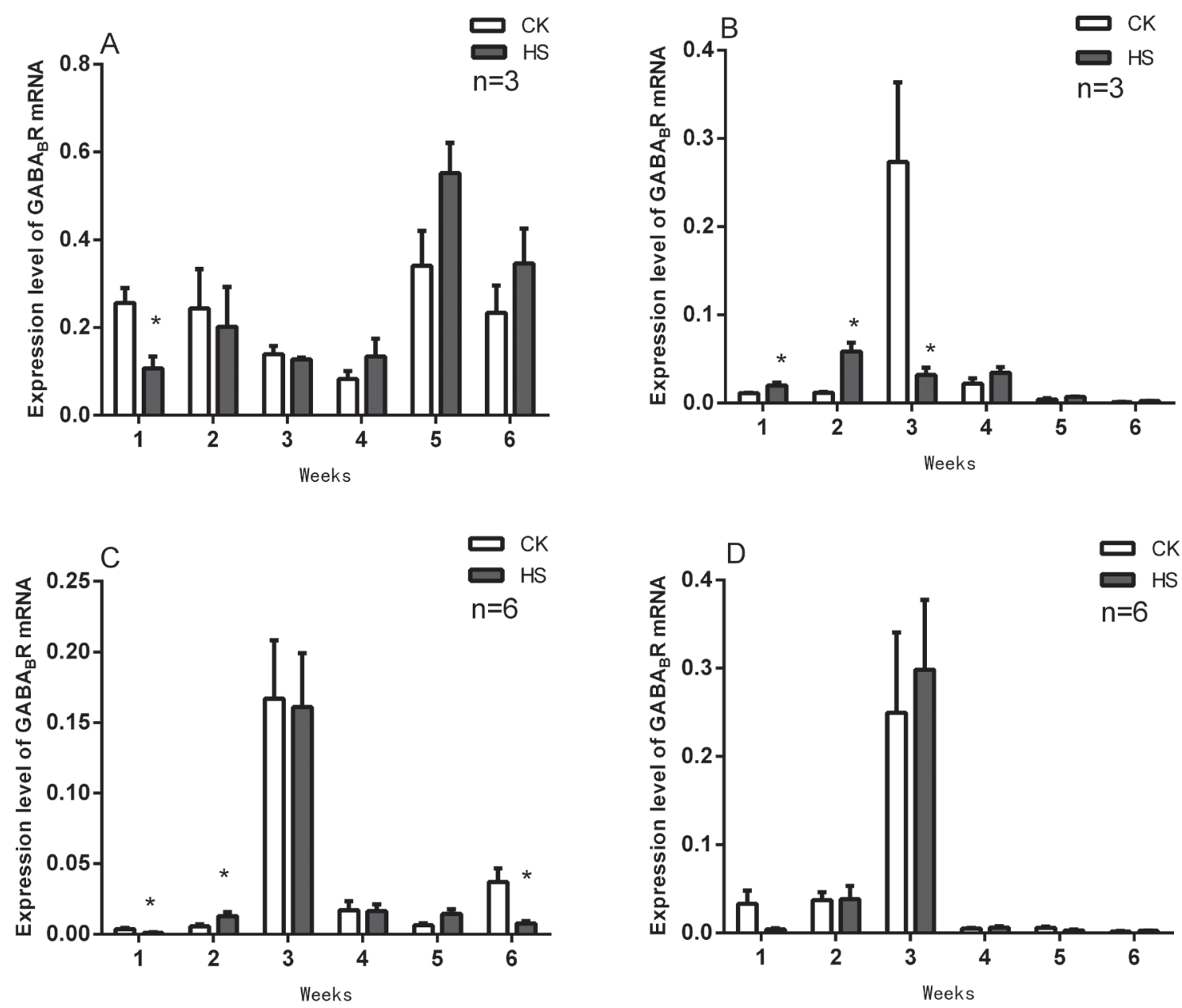

Figure 3 - Relative levels of GABAB receptor mRNA in the hypothalamus, pituitary, testis, and ovary of Wenchang chickens. Note: A, hypothalamus; B, pituitary; C, testis; and $D$, ovary. The results are expressed as mean \pm SEM by $2-\nabla C t$; ${ }^{*} p<0.05$.

expression of receptors in the body was damaged by HS, and the appearance of a stable state thereafter may be a result of the body adaptation to the hot environment. Three weeks after HS, the significantly reduced expressions of both $G_{A B A_{A}}$ receptor and $G A B A_{B}$ receptor in the pituitary may an early reproductive development or may be accompanied by a potential hormone development cycle, which may also induce the compensatory synthesis of a large amount of heatshock proteins (HSPs), leading to a reduction in the production of other proteins (Tu et al., 2010). When HS significantly increased the mRNA expression of the GABA receptor, the latter played a positive regulation role, resulting in an enhanced inhibition of GABAergic neurons. However, when HS significantly reduced the expression of GABA receptor mRNA, the GABA receptor functioned as a negative regulator of HS response, leading to a weakened inhibition. Previous studies have shown that treatment of spermatogonial stem cells with GABA increases the expression of GABA receptor, but inhibits cell proliferation, while treating oocytes with GABA increases the expression of GABA receptor, but reduces estrogen production (Biggs et al., 2013; Du et al., 2013). The animal's body can regulate sexual development and maturation by secreting hormones of the HPG axis, which is the center controlling the development and function of reproductive system in animals. The classic pathway of the HPG axis is as follows: hypothalamus-secreted gonadotropinreleasing hormone $(\mathrm{GnRH})$ reaches the pituitary and stimulates the pituitary gonadotropin (GTH) secreting cells to synthesize and release luteinizing hormone (LH) and follicle-stimulating hormone (FSH); these gonadotropins reach the gonad via circulation and subsequently activate the production of sex hormones, such as testosterone, progesterone, etc. Akinci et al. (1999) showed that multiple $G_{A B A}$ receptor subunits, including the $G A B A_{A}$ receptor $\beta 3$ subunit, are present in the testis and ovary. Upon binding to the GABA receptors, GABA can regulate the synthesis and release of sex and other related hormones in the HPG axis, thereby exerting regulatory effects on the reproductive 
development of animals (Akinci \& Schofield, 1999). Studies also found that, by affecting the nutrient absorption, HS may cause metabolic disorders and altered progesterone levels, resulting in prolonged calving-to-estrus intervals, decreased estradiol levels, reduced generation of ovarian granulosa cells, and decreased vitality of follicles in cows (Rensis \& Scaramuzzi, 2003). The regulatory effects of HS, GABA receptor, and sex hormones on the development of HPG axis are a particularly complex process that warrants further investigations.

In conclusion, the levels of $\mathrm{GABA}_{A}$ receptor and $\mathrm{GABA}_{B}$ receptor mRNAs varied in different tissues of the HPG axis in chickens of different ages, displaying temporal and spatial variations. Heat stress affected the expression of GABA receptor mRNA. Under heat stress, the expression of GABA receptor mRNA is significantly increased or decreased in the different tissues of the HPG axis.

\section{ACKNOWLEDGEMENTS}

This work is supported by research grants from National Natural Science Foundation of China (NSFC 31560680, 31260555).

\section{REFERENCES}

Akazome Y, Abe T, Mori T. Differentiation of chicken gonad as an endocrine organ: expression of LH receptor, FSH receptor, cytochrome P450c17 and aromatase genes. Reproduction 2002;123(5):721-728.

Akinci MK, Schofield PR. Widespread expression of GABA $_{A}$ receptor subunits in peripheral tissues. Neuroscience Research 1999:35(2):145153.

Biggs K, Seidel JS, Wilson A, Martyniuk CJ. $\gamma$-Aminobutyric acid (GABA) receptor subunit and transporter expression in the gonad and liver of the fathead minnow (Pimephales promelas). Comparative Biochemistry and Physiology A: Molecular \& Integrative Physiology 2013;166(1):119127.

Cai YF, Li L, Liu Q, Xu S, Liu H, Wang G. Analysis of apoptosis and expression of bax- $\alpha$ of lymphocytes in peripheral blood of dairy cows. Journal of Nanjing Agricultural University 2004;28(1):66-70.

Chen Z, Wang T, Huang L, Fang D. Effects of GABA on the heat stress broilers. Zoological Research 2002;23(4):341-344.

Chen Z, Xie J, Wang B, Tang J. Effect of $\gamma$-aminobutyric acid on digestive enzymes, absorption function, and immune function of intestinal mucosa in heat-stressed chicken. Poultry Science 2014;93(10):24902500 .

Chen Z, Xie J, Hu MY, Tang J, Shao ZF, Li MH. Protective effects of $\gamma$-aminobutyric acid (GABA) on the small intestinal mucosa in heatstressed WenChang chicken. Journal of Animal and Plant Science 2015;25(1):78-87.
Cheng ZY, Chebib M, Schmid KL. Identification of GABA receptors in chick cornea. Molecular Vision 2012;18:1107-1114.

Du Y, Du Z, Zheng H, Wang D, Li S, Yan Y, et al. GABA exists as a negative regulator of cell proliferation in spermaogonial stem cells. Cellular \& Molecular Biology Letters 2013;18(2):149-162.

Elnagar SA, Scheideler SE, Beck MM. Reproductive hormones, hepatic deiodinase messenger ribonucleic acid, and vasoactive intestinal polypeptide-immunoreactive cells in hypothalamus in the heat stressinduced or chemically induced hypothyroid laying hen. Poultry Science 2010;89(9):2001-2009.

Erdö, SL, Rosdy B, Szporny L. Higher GABA concentrations in fallopian tube than in brain of the rat. Neurochemistry 1982;38(4):1174-1176

Jin Z, Bhandage AK, Bazov I, Kononenko O, Bakalkin G, Korpi ER, et al. Expression of specific ionotropic glutamate and GABA-A receptor subunits is decreased in central amygdala of alcoholics. Frontiers in Cellular Neuroscience 2014;8:288-296.

Jonaidi H, Babapour V, Denbow DM. GABAergic control of food intake in the meat-type chickens. Physiology \& Behavior 2002;76(4-5):465-468.

Li YZ, Cui YQ. Effects of heat stress on reproductive performance, blood reproductive hormone concentrations and gene mRNA expression in different varieties of chicken. Journal of China Agricultural University 2013;18(1):134-141.

McMichael AJ, Dear KB. Climate change: heat, health, and longer horizons. PNAS 2010;107(21):9483-9484.

Rensis FD, Scaramuzzi RJ. Heat stress and seasonal effects on reproduction in the dairy cow. Theriogenology 2003;60(6):1139-1151.

Sohail MU, ljaz A, Yousaf MS, Ashraf K, Zaneb H, Aleem M, et al. Alleviation of cyclic heat stress in broilers by dietary supplementation of manna-noligosaccharide and Lactobacillus-based probiotic: Dynamics of cortisol, thyroid hormones, cholesterol, C-reactive protein, and humoral immunity. Poultry. Science 2010;89(9):1934-1938.

Tu YJ, Chen GH, Geng ZY, Su YJ, Wang KH. HPT Axis HSP70 mRNA dynamic expression during cold stress of goose. Scientia Agricultura Sinica 2010;43(7):1473-1479.

Wang B, Xie J, Chen Z, Lin X, Li ZW. Effect of prenatal heat stress on the development of $\mathrm{GABA}_{B}$ receptor in newborn mouse pituitary. Progress in Veterinary Medicine 2011; 32(6): 60-63.

Wang DQ, Tian Y, Li JJ. Study on HSP70 mRNA expression of different tissues in Shaoxing duck under heat stress. China Poultry 2012;34(6):24-28.

Wang JT, Xu SW. Effect of cold stress on hypothalamic-pituitary-adrenal axis in chicken. Veterinary Science in China 2007;37(7):614-618.

Xie YZ, Xie J, Chen Z, Tang J, Li ZW, Zhang JH. Impact of prenatal heat stress on the development of GABA receptors in the genital gland of newborn mice. Veterinary Science in China 2011;41(3):298-302.

Yin F, Chen Z, Li ZW, Tang J. Influence of acute heat stress on the development of GABAergic neurons in HPA-axies of mouse embryos. Journal of Thermal Biology 2011;36(8):486-491.

Zhao YJ, Xu SY, Jiang RS. Expression analysis of SFRP1 and PDK4 gene in livers of chicken under heat atress. China Poultry 2012;34(15):12-15. 